WHO. To evaluate current algorithms for treatment of gonorrhoea, chlamydial infection and trichomoniasis, we conducted laboratory testing with vaginal specimens from women presenting with cervicitis or vaginitis syndromes at a sexually transmitted infections clinic in Kingston and at follow-up to assess cure rates for these infections.

Methods From August, 2010 through January, 2011, vaginal swab specimens were obtained from 258 women $>18$ years old during a routine clinical examination prior to syndromic treatment according to local guidelines. Treatment for gonorrhoea, chlamydial infection and trichomoniasis was prescribed for women with cervicitis. Treatment for trichomoniasis, bacterial vaginosis and candidiasis was prescribed for women with abnormal vaginal discharge. Women returned the next week for follow-up assessment and specimen collection. Specimens were tested for Neisseria gonorrhoeae (NG) and Chlamydia trachomatis (CT) using APTIMA Combo2 and for Trichomonas vaginalis (TV) using APTIMA analyte-specific reagents. All baseline specimens were tested. Follow-up specimens from women with a positive baseline test or who remained symptomatic were also tested. Patients with a positive follow-up test were contacted and instructed to return to the clinic for additional treatment. Cure was defined as a positive baseline test and a negative follow-up test.

Results Baseline prevalence of infection with NG was $11.7 \%$, CT was $20.7 \%$, TV was $25.6 \%$. At least one of these STIs was detected by laboratory testing in $40.7 \%$ of women. Co-infections were common. Women with TV were more likely to have NG or CT than women without TV (OR: 2.6, 95\% CI 1.4 to 4.8). STI testing at follow-up indicated cure rates of $77.3 \%$ for NG, $43.5 \%$ for CT and $47.1 \%$ for TV infections. CT incidence at follow-up was $5.9 \%$; no incident NG or TV infections were detected.

Conclusions With syndromic management, just over half of the STIs in women that were detected by laboratory testing at baseline were cured at follow-up. Reinfection, incorrect or inadequate treatment, failure to comply with treatment instructions or treatment failure could potentially explain prevalent STIs that were detected at follow-up. The low cure rates for chlamydial infection and trichomoniasis are cause for concern.

\section{P3-S1.18 SIDE EFFECTS OF DOXYCYCLINE IN ADOLESCENTS TREATED FOR PELVIC INFLAMMATORY DISEASE}

\section{doi:10.1136/sextrans-2011-050108.418}

${ }^{1}$ W Risser, ${ }^{2} \mathrm{~J}$ Risser. ${ }^{1}$ University of Texas, Medical School at Houston, Houston, USA; ${ }^{2}$ University of Texas, School of Public Health, USA

Background Our purpose was to assess adverse reactions to doxycline in adolescents under treatment for PID.

Methods At the Harris County (Houston), Texas, USA Juvenile Detention Center from May 2008 through January 2010, we evaluated adolescents in whom we diagnosed PID. We used the diagnostic criteria and outpatient antibiotic regimen for PID recommended by the CDC. We gave $250 \mathrm{mg}$ ceftriaxone intramuscularly once, and, if they were not pregnant, vomiting, or ill enough to also require metronidazole, we prescribed doxycline, $100 \mathrm{mg}$ twice a day for 14 days. The doxycycline was given before, during, or after breakfast, and approximately $2 \mathrm{~h}$ after supper. With the medicine, patients were routinely given a packet of two crackers. We re-assessed these patients at $48-72 \mathrm{~h}$ and again at least at 7 and 14 days. At each follow-up, we discussed side effects of doxycycline. Results We evaluated 141 consecutive patients in whom we made the diagnosis of mild to moderate PID: 55\% were black, 31\% Hispanic, and $15 \%$ white. The mean age (SD) was $15.4(1.2)$ years. Of the 141 patients, $86(61 \%)$ had no problems when the doxycline was given with two crackers. Forty-five (32\%) required more substantial food to avoid nausea and/or vomiting: the medication was given immediately after breakfast or with a sandwich after supper. Those who had side effects in the morning had usually skipped breakfast. Ten (7\%) complained of significant gastrointestinal side effects that were not relieved by the simultaneous ingestion of food, and we gave a dose of promethezine $1 \mathrm{~h}$ before the doxycycline.

Conclusion $39 \%$ of our patients required more than a small amount of food (two crackers) to avoid gastrointestinal side effects from doxycline. Our results suggest that clinicians should advise adolescents to take this antibiotic after eating a greater amount of food if a small snack does not prevent this problem. Because some patients (7\%) did not tolerate doxycline even after they ate a meal or a sandwich, we also recommend early contact with patients who have PID to assess the need to change medications or to add an anti-emetic.

\section{P3-S1.19 EVALUATION OF THE COBAS ${ }^{\circledR} 4800$ CT/NG TEST USING CLINICIAN AND SELF-COLLECTED VAGINAL SWABS, CERVICAL SPECIMENS IN PRESERVCYT SOLUTION, AND PHARYNGEAL THROAT WASH SPECIMENS}

doi:10.1136/sextrans-2011-050108.419

M Krevolin, A Sunhachawee, D Hardy, J Pane, E Cervantes, R Kotadia, S Joshi, R Puckett, 0 Liesenfeld. Roche Molecular Systems, Pleasanton, USA

Objective To evaluate the limit of detection (LOD), inclusivity, exclusivity, and interfering substances of the cobas ${ }^{\circledR} 4800 \mathrm{CT} / \mathrm{NG}$ Test using vaginal, PreservCyt, and pharyngeal specimens.

Methods Chlamydia trachomatis (CT) and Neisseria gonorrhoeae (NG) cultures were diluted into pools of negative patient specimens at six concentrations to determine the LOD (lowest concentration giving $a \geq 95 \%$ hit rate). A panel of 15 serovars of CT, plus the Swedish variant (nvCT), and 45 strains of NG was diluted into each sample matrix to determine inclusivity. To ensure specificity, a panel of 184 non-CT and non-NG organisms that may be found in the oral or urogenital region was tested at $\dot{\mathrm{G}} \geq 10^{\wedge} 5 \mathrm{CFU}$ or copies $/ \mathrm{ml}$. The organisms were diluted into CT/NG positive and negative samples. Ten CT/NG positive and negative samples were spiked with blood (up to $5 \%$ ), leukocytes (up to $10^{\wedge} 7$ cells $/ \mathrm{ml}$ ), cervical mucus, and saliva at concentrations up to $10 \%$ to check for interference. Ten (for pharyngeal) and 18 (for urogenital) over-the-counter products were also tested.

Results LODs were as follows: Vaginal CT $10 \mathrm{IFU} / \mathrm{ml}$, NG $100 \mathrm{CFU} / \mathrm{ml}$; PreservCyt CT $0.6 \mathrm{IFU} / \mathrm{ml}$, NG $3.5 \mathrm{CFU} / \mathrm{ml}$, Pharyngeal CT $0.5 \mathrm{IFU} / \mathrm{ml}, \mathrm{NG} 2.25 \mathrm{CFU} / \mathrm{ml}$. All 15 serovars of CT, plus the Swedish variant (nvCT), and 45 strains of NG were detected at, or near the LOD. No cross reactivity was noted with 184 non-CT and non-NG organisms in any specimen type. Vaginal but not PreservCyt samples showed interference from leukocytes at $>10^{\wedge} 6 \mathrm{cell} / \mathrm{s} / \mathrm{ml}$. This interference was noted as clot detections and samples were not processed. Pharyngeal samples showed false negative results in the presence of blood at $>0.25 \%$, and saliva at $>2.5 \%$. No interference was noted with any over-the-counter products in any specimen type.

Conclusions Vaginal, PreservCyt, and pharyngeal specimens are suitable sample types for the cobas ${ }^{\circledR} 4800$ CT/NG Test.

\section{P3-S1.20 AETIOLOGY OF URETHRAL DISCHARGE SYNDROME AND ITS ASSOCIATION WITH SEXUAL PRACTICES AMONG MALES ATTENDING STI CLINICS IN INDIA}

doi:10.1136/sextrans-2011-050108.420

${ }^{1} \mathrm{R}$ Kumar, ${ }^{2} \mathrm{P}$ Parimi, ${ }^{1} \mathrm{P}$ Narayanan, ${ }^{1} \mathrm{~A}$ Das, ${ }^{1} \mathrm{~A}$ Gurung, ${ }^{3} \mathrm{G}$ Rao, ${ }^{3} \mathrm{~A}$ Risbud. ${ }^{1}$ Family Health International, New Delhi, India; ${ }^{2}$ HIV AIDS Alliance, Hyderabad, India; ${ }^{3}$ National AIDS Research Institute, Pune, India

Background Studies to validate the aetiology of urethral discharge (UD) syndrome are limited in India. The objectives of the study 